\title{
GCU
}

Glasgow Caledonian

University

University for the Common Good

\section{Loneliness in adolescence: a Rasch analysis of the Perth A-loneness Scale}

Houghton, Stephen; Marais, Ida; Hunter, Simon C.; Lawrence, David; Tan, Carol; Carroll, Annemaree

Published in:

Quality of Life Research

DOI:

$10.1007 / \mathrm{s} 11136-020-02635-\mathrm{x}$

Publication date:

2021

Document Version

Author accepted manuscript

Link to publication in ResearchOnline

Citation for published version (Harvard):

Houghton, S, Marais, I, Hunter, SC, Lawrence, D, Tan, C \& Carroll, A 2021, 'Loneliness in adolescence: a Rasch analysis of the Perth A-loneness Scale', Quality of Life Research, vol. 30, no. 2, pp. 589-601.

https://doi.org/10.1007/s11136-020-02635-x

\section{General rights}

Copyright and moral rights for the publications made accessible in the public portal are retained by the authors and/or other copyright owners and it is a condition of accessing publications that users recognise and abide by the legal requirements associated with these rights.

Take down policy

If you believe that this document breaches copyright please view our takedown policy at https://edshare.gcu.ac.uk/id/eprint/5179 for details of how to contact us. 
Houghton, S., Marais, I., Hunter, S. C., Hattie, J., Carroll, A., Lawrence, D., \& Tan, C. (in press). A Rasch analysis of the Perth A-loneness Scale. Quality of Life Research.

This is the final Author Accepted Manuscript. 


\begin{abstract}
Purpose

The psychometric properties of the Perth A-loneness Scale (PALs) have been extensively validated using classical test theory, but to date no studies have applied a Rasch analysis. The purpose of this study was to validate the PALs four subscales, using Rasch analysis.
\end{abstract}

\title{
Methods
}

Responses from 1,484 adolescents (58\% female, mean age $=12.8$ years), 131 of whom had a diagnosed neurodevelopmental disorder, from 10 Western Australian secondary schools were included in the Rasch analysis. Overall fit, individual item fit, local response dependence, dimensionality, operation of response categories, and differential item functioning (DIF) were examined.

\section{Results}

The Rasch analysis supported the factor structure of the PALs. A reasonable to high reliability was obtained for each of the subscales. Participants did not distinguish consistently between the higher categories 'very often' and 'always' on three of the subscales. No item showed Differential Item Functioning (DIF) for neurodevelopmental disorder status and age. One item on each of the Positive and Negative Attitude to Aloneness subscales showed DIF for gender.

\section{Conclusion}

The results support the interval scale measurement properties of the PALs and provides clinicians and researchers with a measure to assess adolescent loneliness, a construct strongly associated with a constellation of mental health problems.

Keywords: Rasch model, Loneliness, Adolescents, Psychometrics 


\section{Introduction}

Loneliness is a debilitating psychological condition characterised by a deep sense of social isolation, emptiness, worthlessness, lack of control, pain and personal threat [1]. It can even manifest among those surrounded by many people or with numerous contacts, followers, or friends on social media [2]. Definitions of loneliness converge on a construct that is a distressing emotional state people experience when they notice a discrepancy in the desired and perceived quality or quantity of their social relations [3, 4].

Although $80 \%$ of adolescents experience feelings of loneliness at some time, approximately 22\% experience it in a chronic, persistent, and even pathological form [5]. This is clinically relevant because of the associations between loneliness and mental health problems e.g., anxiety and depression [6]. Moreover, an increasing loneliness trajectory predicts social withdrawal, self-harming, suicide ideation [7] and more lethal suicide attempts [8].

The social communication difficulties that adolescents with Neurodevelopmental Disorders (NDDs e.g., Specific Learning Disorders, Autism Spectrum Disorder, Attention Deficit Hyperactivity Disorder) [9] experience significantly limit both the number and quality of their friendships, which increases their risk of feeling lonely [9, 10]. Being especially prone to loneliness $[11,12]$ is clinically relevant because loneliness increases the risk of developing additional mental health problems [13].

Loneliness has been conceptualised and measured as both a unidimensional and multidimensional construct. Among the measures used to evaluate loneliness, the 20 item Revised University of California at Los Angeles Loneliness Scale (R-ULS) is on e of the most commonly used [14]). The R-ULS was recognized as having a two-dimensional construct, for which the second one contributed to a methodologic artefact related to negatively worded items. Thus, shortened versions of R-ULS were developed, including 
11 -item [15]), 8 - item [16, 17]), 6-item $[18,19]$ and three-item vers ions [20]. These successive versions of the scale have theoretically thought of loneliness as a single dimension, although the factor structure has been found to vary between one and three factor solutions. The 11-item de Jong Gierveld Loneliness Scale [DJGLS, 21] also taps a single dimension of loneliness. However, with both the R-ULS and DJGLS “ambiguity” and “controversy” continue regarding their dimensionality [22, 23].

However, the multidimensional approach has become more widely accepted and dominant $[24,25]$ and several researchers have provided strong evidence that loneliness is a multidimensional construct $[1,4,26,27]$. Important distinctions have been forthcoming via the multidimensional measurement approach, including, for example, between loneliness and aloneness, and that attitudes toward being alone might affect one's vulnerability to feeling lonely when alone [27]. Few instruments for adolescents reflect this distinction, however.

The multidimensional approach assumes that loneliness can vary in intensity and across causes and circumstances, and where different social relationships give rise to different forms of loneliness. One of the most widely used multidimensional measures of loneliness is The Loneliness and Aloneness Scale for Children and Adolescents (LACA; Marcoen et al., 1987 [28]). This comprises 48-items spread over four subscale (12 items each) that capture family loneliness, peer-related loneliness, aversion to aloneness, and affinity for aloneness (see Maes et al., 2015 [29]). Another multidimensional measure is The Perth A-loneness scale [1], a 24item self-report measure comprising four factors (each with 6 items) that measure quality of friendships, feelings of isolation, positive attitudes towards aloneness, and negative attitudes towards aloneness. Unlike other measures the PALs is based on extensive interviews conducted with adolescents, was developed specifically for adolescents from metropolitan and rural environments, measures positive aspects of loneliness, and does not include family related items because at this time peers become more prominent in the lives of young people. 
Like other multidimensional measures of loneliness, the PALs has applied Classical Test Theory (CTT) in testing the validity of the construct and the reliability of its scores.

Confirmatory Factor Analyses have established four correlated factors with satisfactory model fit and strong internal reliabilities for each subscale: [1, 30, 31]. However, additional information above using CTT can be obtained using an Item Response Theory model. In the application of modern test theory two paradigms have emerged: a statistical modelling paradigm (IRT) and an experimental measurement paradigm (Rasch Measurement Theory RMT) [32]. In the present study, the psychometric properties of each of the subscales of the PALs was examined for fit to the Rasch model [33, 34].

The Rasch Model (as used within the RMT paradigm) has a number of advantages [see 32, 35]. Essential information about measurement equivalence, irrespective of groups (e.g., cultural) or the ages and genders within these groups, can also be examined via Differential Item Functioning (DIF) [36]. These properties (of the Rasch model) ensure that the empirical ordering of the PALs response categories is as required. Also, because the total score for each subscale is a sufficient statistic for the trait values, this ensures that all those with the same total score will get the same estimated trait value. This permits valid comparisons to be made between different groups on each of the subscales.

By considering both psychometric and item content, as well as the theoretical relevance of the items in each of the four PALs subscales, along with confirming that any DIF is real (and not artificial) the present study evaluated the psychometric properties of the construct and the reliability of each of the PALs four subscales.

\section{Method}

\section{Participants and settings}

The sample comprised 1,484 10-17 years olds (607 male, 861 female, 16 missing) from Grades 5 to 10 (Mean age $=12.8$ years, SD $=1.4$ ) in 10 Western Australian mainstream 
schools. Of the sample, 131 had received a diagnosis from a paediatrician as meeting DSMIV-TR [37] criteria for Attention-Deficit Hyperactivity Disorder, Autism Spectrum Disorder [Level 1, social difficulties that require some support], and/or Specific Learning Disorders (i.e., NDDs).

Of the 10 participating schools, 6 were state government secondary schools, 3 were nongovernment schools $(\mathrm{K}-12)$ and 1 was a state government primary school (10 - 11 year olds). All participating schools were located across a range of socio-economic areas as indexed by their Index of Community Socio-Educational Advantage (ICSEA). ICSEA is set at an average of $1000(S D=100)$ and the higher the ICSEA value, the higher the level of educational advantage of students who go to this school (and vice versa). The three nongovernment secondary schools recorded the highest ICSEA values (range 1044 to 1149). Of the six state government secondary schools, four had ICSEA values ranging from 866 to 988, and one was 1009. The state government primary school ICSEA was 934.

\section{Instrument}

\section{The Perth A-loneness scale (PALs) [1]}

The Perth A-loneness scale [1] is a validated 24-item self-report measure of adolescent loneliness, comprising four correlated factors, each with 6 items. Factor One measures quality of friendships (e.g., My friends will stand by me in almost any difficulty); Factor Two, feelings of isolation (e.g., I feel like I do not have a friend in the world); Factor Three, positive attitudes towards being alone (e.g., I have discovered the benefits of being alone); and Factor Four, negative attitudes towards being alone (e.g., When I am all by myself, I wish I had a friend to be with). Participants respond using a six point Likert scale: 1 = never, 2 = rarely, 3 = sometimes, 4 = often, 5 = very often, 6 = always. Previous research has revealed significant correlations between the four factors [1, 31]. Delivered online, its items provide a maximum total score of 36 for each of the four subscales. 


\section{Procedure}

Permission to conduct the research was obtained from The Human Research Ethics Committees of The University of Western Australia and the Western Australian Department of Education. Fifteen schools across a range of socioeconomic areas were randomly selected and information sheets about the research were sent to the school principals. Ten schools agreed to be involved. Letters of introduction, along with information sheets and consent forms were forwarded to the parents of students in school grades 5, 7 and 9. Informed consent was obtained from individual participants included in the study. School principals nominated one teacher to liaise with the researchers and to administer the online survey. These teachers each received written instructions to ensure standardisation of administration. A response rate of $73 \%$ was obtained and these students completed the PALs survey during regular school hours. The electronic survey remained open for four weeks.

\section{Statistical analysis}

Responses to each of the four PALS subscales (i.e., friendship related loneliness, isolation, positive attitude to aloneness, and negative attitude to aloneness) were analysed separately according to the polytomous Rasch model (partial credit parameterization of the model) [38] using the Rasch Unidimensional Measurement Model (RUMM2030) software [39]. The following aspects of Rasch analysis were assessed:

\section{Person/Item alignment and reliability}

An estimate of the reliability of the scale is available in RUMM2030 as a person separation index (PSI). Values range between 0 and 1, which reflect the instrument's capability to differentiate persons on the continuum. Where there is no appreciable missing data or when the distribution of scores are skewed then the PSI is analogous to Cronbach's coefficient alpha [40]. The threshold between two adjacent response categories is the point on 
the measurement continuum where the probability of a response in either of the two adjacent response categories is equal. Histograms of the Rasch person estimates against the item threshold estimates on the same scale indicate how items 'difficulty to endorse' align with the estimates of the persons.

\section{Fit to the Rasch model}

Item fit is assessed individually for each item as well as by summary statistics. Item Characteristic Curves (ICCs) show graphically how an item fits with what was expected according to the model. Statistical item misfit was examined using the item fit-residual statistic and the item chi-square fit statistic [41]. Items with values on these two statistics that are large, relative to the values of the other items, indicate misfit. The total item-trait interaction chi-square, which is the sum of the individual item chi-square statistics, shows whether the items fit the model overall.

Superimposed on the person/item threshold distribution (see Figures 1 to 4 ) the information function (in green) provides a visual representation of the amount of information provided by the scale at different levels of the trait being measured. The space below the line represents the amount of information provided, with greater information indicating greater measurement precision (less measurement error).

Unidimensionality was assessed through a Principal Components Analysis (PCA) of the residuals as well as through the fit statistics. Unidimensionality is supported when the variance explained by the first principal component is small, usually indicated by an eigenvalue size $<2.0$ [42]. Items with residual correlations greater than the average correlation are taken as locally dependent and thus redundant.

\section{Operation of response categories}

Item thresholds that are not ordered sequentially indicate some problem with the empirical ordering of the categories [32]. Category Characteristic Curves (CCCs) show the functioning 
of the response categories and identify where response categories are not functioning as expected.

\section{Group differences at the item level and group means}

Rasch analysis allows diagnosis of differential item functioning (DIF) for various subgroups of the population. An item shows DIF when, for the same level of the trait being measured, members of one sub-group (e.g., males) score differently on the item than members of another sub-group (e.g., females). Many procedures have been proposed for detecting DIF including the Mantel-Haenszel procedure, methods based on logistic regression analysis and other procedures, like those used in Rasch analysis. Most DIF methods demonstrate similar results. In a recent study [43] all three methods that were applied (the Mantel-Haenszel procedure, Logistic regression, and Rasch analysis) "led to similar findings regarding the presence of DIF. There was remarkable consistency between the methods in the size and direction of the DIF effects found” (p.2885).

In the present study, DIF is diagnosed through an inspection of the ICC and is confirmed statistically through an analysis of variance (ANOVA) of the residuals [41]. Items were tested for DIF for gender, age (early adolescents 10-12 years and adolescents 13+ years), and NDDs (diagnosed/not diagnosed).

\section{Results}

The sample comprised of 1,484 adolescents from school grades 5 (10 years of age) through to $11(16 / 17$ years) $($ Mean age $=12.8$ years, $S D=1.4)$. Of the sample, 98 were aged 10 years, 192 aged 11 years, 309 aged 12 years, 411 aged 13 years, 325 aged 14 years, 141 aged 15 years, and 5 were aged 16/17 years. All respondents who had missing responses in a subscale were deleted from the analysis of that particular subscale, leaving 1,452 in the Friendship Related Loneliness subscale, 1,448 in the Isolation subscale, 1,433 in the Positive Attitude to Aloneness subscale and 1,441 in the Negative Attitude to Aloneness subscale. Of the sample, 528 were classified as early adolescents $(10-12$ years, mean age $=11.35, \mathrm{SD}=$ 
0.75), 830 were adolescents $(13-17$ years, mean age $=13.71, \mathrm{SD}=0.75)$ and 3 did not specify their age.

\section{Friendship Related Loneliness Subscale}

Reliability and Item/Person alignment: Figure 1 (top) shows how the items and respondents aligned for the Friendship Related Loneliness subscale, the histogram in red shows the Rasch person estimates and the histogram in blue shows the item threshold estimates. The mean of the person estimates was $1.35(\mathrm{SD}=1.54)$. Relative to the item thresholds, which have a constrained mean of 0 , the person mean is positive, indicating that, on the whole, respondents mostly responded with often, very often and always to the items in the subscale. It is clear from the figure that most respondents had large positive estimates. The scale is scored so that higher values indicate higher levels of friendship quality and lower levels of loneliness, thus suggesting that a majority of adolescents in this sample had good friendships and low levels of loneliness. Because of the negatively skewed distribution, the PSI (0.84) and Cronbach's coefficient alpha (0.91) did not have equivalent values. However, both indicate very good reliability.

Figure 1 here

Item fit: The overall Friendship Related Loneliness item/trait chi-square statistic value $\left(\chi^{2}=\right.$ 19.77; $\mathrm{df}=24 ; \mathrm{p}<0.71)$ indicated that the items fit the model. No items misfit according to more than one fit statistic and the Item Characteristic Curves, which showed good fit graphically, confirmed the lack of misfit. Item fit and the item residual correlation matrix showed that two items were locally dependent. Item 15 (I get plenty of help and support from friends) and item 16 (My friends will stand by me in almost any difficulty) had a positive item residual correlation that was large $(0.081)$ relative to the average correlation, which was negative (-0.194) due to the small number of items [41]. 
Considering the meaning of the two items appear to be quite similar, one of the items could be considered redundant and be replaced with an item that measures a more unique aspect of the construct. The PCA of the residuals confirmed the unidimensionality of the subscale. The eigenvalues of the first and second principal components were 1.543 and 1.349 respectively.

Operation of response categories: Thresholds for all items were ordered sequentially, indicating that the response categories functioned as intended. Figure 1 (bottom) shows the CCC for item 1 (I feel part of a group of friends). It is clear from the graph that each response option has a turn at being the most probable response, and that they are in order.

\section{Isolation subscale}

Reliability and Item/Person alignment: Three hundred and ten respondents had extreme scores on this subscale, that is, they responded with Never on all of the items. Figure 2 (top) shows how the items and persons aligned for the Isolation subscale. The mean of the person estimates was $-1.49(\mathrm{SD}=1.30)$. Relative to the item thresholds, which have a constrained mean of 0 , the person mean is negative. That is, overall persons responded never, rarely and sometimes to the Isolation items, indicating they do not feel isolated. Because of the very positively skewed distribution, the PSI (0.67) and Cronbach’s coefficient alpha (0.83) did not have equivalent values. Alpha indicated very good reliability, whereas the PSI indicated reasonable reliability.

Figure 2 here

Item fit: The overall Isolation item/trait chi-square statistic value $\left(\chi^{2}=40.62 ; \mathrm{df}=24 ; \mathrm{p}<\right.$ 0.02) indicated that there may be some items that do not fit the model. However, no items misfit according to more than one fit statistic and the Item Characteristic Curves, which showed good fit graphically, indicated a lack of misfit. Item fit and the item residual correlation matrix indicated no dependent items. The PCA of the residuals confirmed the 
unidimensionality of the subscale. The eigenvalues of the first and second principal components were 1.477 and 1.237 respectively.

Operation of response categories: Thresholds for all items were disordered, indicating that the response categories did not function as intended. Figure 2 (bottom) shows the CCC for item 14 (No one cares much about me). It is clear from the graph that each response option does not have a turn at being the most probable response, thereby indicating that the higher response categories are not functioning as intended. In particular, participants did not respond consistently to 4 (very often) and 5 (always). This pattern was evident in all of the items.

\section{Positive Attitude to Aloneness subscale}

Reliability and Item/Person alignment: Figure 3 (top) shows how the items and persons aligned for the Positive Attitude to Aloneness subscale. Unlike the Friendship Related Loneliness and Isolation subscales, the distribution of person estimates was normal. The mean of the person estimates was $-0.056(\mathrm{SD}=1.114)$, which aligned well with the items (mean $=0)$. The PSI (0.83) and Cronbach’s coefficient alpha (0.84) had equivalent values, indicating very good reliability.

Figure 3 here

Item fit: The overall Positive Attitude to Aloneness item/trait chi-square statistic value $\left(\chi^{2}=\right.$ 30.91; $\mathrm{df}=24 ; \mathrm{p}<0.16$ ) indicated that the items fit the model. No items misfit according to more than one fit statistic and the Item Characteristic Curves, which showed good fit graphically, confirmed the lack of misfit. Item fit and the item residual correlation matrix showed that two items were dependent. Item 18 (I have discovered the benefits of being alone) and item 20 (There are benefits of being on my own) had a positive item residual correlation that was large $(0.045)$ relative to the average correlation, which was negative (0.190) due to the small number of items [41].

Considering that the wording of the two items is quite similar, one of the items could be considered redundant and can be replaced with an item that measures a more unique aspect of 
the construct. The PCA of the residuals confirmed the unidimensionality of the subscale. The eigenvalues of the first and second principal components were 1.612 and 1.271 respectively.

Operation of response categories: As with the Isolation subscale, the thresholds for all items were disordered. Figure 3 (bottom) shows the CCC for item 11 (There are positive things about being alone), which was representative of all the items. Respondents did not respond consistently to 4 (very often) and 5 (always).

\section{Negative Attitude to Aloneness subscale}

Reliability and Item/Person alignment: Figure 4 (top) shows how the items and persons aligned for the Negative Attitude to Aloneness subscale. Similar to the Positive Attitude to Aloneness subscale, the distribution of person estimates was normal. The persons (Mean $=-$ 0.222 , SD $=0.84)$ aligned well with the items (mean $=0)$. The PSI $(0.76)$ and Cronbach's coefficient alpha (0.77) had equivalent values, and indicated satisfactory reliability.

Figure 4 here

Item fit: The overall Negative Attitude to Aloneness item/trait chi-square statistic value $\left(\chi^{2}=25.45 ; \mathrm{df}=24 ; \mathrm{p}<0.38\right)$ indicated that the items fit the model. No items misfit according to more than one fit statistic and the Item Characteristic Curves, which showed good fit graphically, confirmed the lack of misfit. Item fit and the item residual correlation matrix indicated no dependent items. The PCA of the residuals confirmed the unidimensionality of the subscale. The eigenvalues of the first and second principal components were 1.549 and 1.388 respectively.

Operation of response categories: Similar to the Positive Attitude to Aloneness subscale, the thresholds for all items in the Negative Attitude to Aloneness subscale were disordered. Figure 4 (bottom) shows the CCC for item 5 (When I am by myself I feel lonely), which was representative of all items. Respondents did not respond consistently to 4 (very often) and 5 (always). 


\section{Differential Item Functioning}

To test for age differences the sample was divided into early adolescents ( $\mathrm{N}=528$, aged 10-12 years) and adolescents ( $\mathrm{N}=830$, aged $13-17$ years); 3 did not specify their age. No item showed DIF for Age. Early adolescents had a higher mean estimate on the Friendship Related Loneliness subscale than adolescents $(\mathrm{F}(1,1447)=12.91, \mathrm{p}=0.001)$. Adolescents had a higher mean estimate on the Isolation subscale than early adolescents $(\mathrm{F}(1,1443)=$ 9.89, $\mathrm{p}=0.002$ ). Adolescents also had a higher mean on the Positive Attitude to Aloneness subscale than early adolescents $(F(1,1428)=34.73, p=0.001)$. There was no difference in means on the Negative Attitude to Aloneness subscale (early adolescents mean $=-0.260$; adolescents mean $=-0.199)$.

No item showed DIF for gender on the Friendship Related Loneliness and Isolation subscales. In addition, the means did not differ significantly according to gender on either of these two subscales. As shown in Figure 5, Item 3 (I feel calm when I'm by myself) on the Positive Attitude to Aloneness subscale showed DIF for gender, with males with the same overall attitude to loneliness scoring higher on this item than females. As an indication of the size of the DIF, the difference in item location between male and female items is 0.51 logits when the items are split according to gender. The group means did not differ significantly $($ male mean $=-0.145$; female mean $=0.002)$.

\section{Figure 5 here}

As shown in Figure 6, item 19 (When I get bored I'm unhappy) on the Negative Attitude to Aloneness subscale showed DIF for gender. Males with the same overall attitude to loneliness scored higher on this item than females. As an indication of the size of the DIF, the difference in item location between male and female items is 0.31 logits when the items are split according to gender. Despite the DIF favouring males on this item, females had a higher mean estimate on the Negative Attitude to Aloneness subscale overall $(F(1,1423)=26.72$, $\mathrm{p}$ $=0.001)$. 
Figure 6 here

No item showed DIF for neurodevelopmental disorder status. It is noted, however, that the sample size of the neurodevelopmental group $(n=131)$ was considerably smaller than the group without a disorder. Ideally, the groups should be approximately equivalent in size for a DIF analysis [41]. There were no differences in the means of respondents with or without a neuro-developmental disorder.

\section{Discussion}

The primary aim of this research was to evaluate the psychometric properties of the four subscales of the Perth A-loneness scale among Western Australian adolescents through the application of the Rasch model. The four PALs subscales were analysed separately according to the polytomous Rasch model. The importance of studying both persons and items in psychometric instruments using Rasch was highlighted as a way of improving validation procedures [44].

All four of the PALs subscales exhibited satisfactory to excellent reliability, which is consistent with previous research applying Classical Test Theory (CTT) [1, 30, 31] to the PALs. The present results provide evidence to support the interval scale measurement properties of the 24-item PALs that covers loneliness and aloneness and which meets the Rasch model criteria. Thus, the measure can discriminate among groups of respondents whose levels of loneliness and aloneness are different.

Of the four PALs subscales, Friendship Related Loneliness was best performing in terms of its psychometric properties, with high reliability, and response categories that functioned as intended. Conversely, the ordering of the categories in the other three PALs subscales were not working as intended. Participants had difficulty in discriminating between the response options "very often" and "always" and therefore future research using the PALs with Australian adolescents should consider these categories for amalgamation into one category. This approach may be a way of enhancing participant clarity regarding what is inferred by 
their response choices, enhancing the measure's parsimony, and consequently reducing participant response burden by removing redundant response choices [45].

In the present study two items in the Friendship Related Loneliness subscale were locally dependent (i.e., item 15 I get plenty of help and support from friends and item 16 My friends will stand by me in almost any difficulty), suggesting one could be deleted. However, the Friendship Related Loneliness subscale measures quality of friendships and the two items in question represent two different facets of this. Friends may provide help and support in general, but not all friends stand by an individual when difficulties arise. Therefore, both items should be included in future administrations. In arriving at this recommendation, we did not rely solely on statistically defined procedures as the basis for deleting items. Rather as recommended [46], the item content and its theoretical relevance to the construct were carefully examined prior to any decision to remove items. In doing so, a more authentic assessment of the latent construct was retained. DIF is a certain form of item bias that can occur when different groups within a sample (e.g. males and females), despite equal levels of the underlying trait, respond differently to an item. No items in the four separate subscales showed DIF by age or NDDs status. The latter construct is important because approximately $20 \%$ of young people have NDDs, with prevalence rates increasing [47]. The social communication difficulties they experience contribute to greater levels of loneliness and consequently a greater risk of mental health problems compared to their peers $[9,13]$. The PALs is equally appropriate for assessing loneliness in those with NDDs and, as such, it responds to calls for loneliness in young people with NDDs to be investigated [9, 48, 49]. Conversely, two items showed DIF for gender. For the same level of Positive Attitude to Aloneness, males endorsed feeling calm when alone more than females. Also for the same level of Negative Attitude to Aloneness males endorsed feeling unhappy when they're bored more than females. 
It should be noted that the present study examined the fit of each of the four subscales of the PALs to the Rasch model. As demonstrated in previous research with the PALs using CTT $[1,30,31]$ these four subscales are not organised together by a higher-order construct. Rather, the PALs has, like other research [4], found distinct groups of adolescents, each with unique profiles of loneliness and positive and negative attitudes towards being alone.

Cultural variations in the experience of loneliness in different countries has been of interest to a number of researchers, particularly between individualistic (i.e., Western or European) cultures and collectivistic (i.e., Eastern or Asian) cultures. For example, using data from separate waves of the European Social Survey of older adults (60 to 85 years or older) and adolescents (aged > 14 years), researchers [50] reported systematic cultural differences in the frequency of experienced loneliness and in predictors of loneliness. Data pertaining to the cultural backgrounds of the adolescents in the present study was not collected and this should be an important area for future investigations.

A large sample of Western Australian male and female adolescents from government and non-government secondary schools, located across a range of socio-economic status areas, and representing a wide age range was recruited. Therefore, we believe that our findings are generalizable to adolescents throughout other states of Australia. However, there was only a relatively small number $(n=131)$ of adolescents with a NDD. The category NDDs comprises different conditions (e.g., Specific Learning Disorders, Autism Spectrum Disorder, and Attention Deficit Hyperactivity Disorder) and feelings of loneliness may differ across these. The smaller sample size prohibited an analysis according to different NDDs and future research should address this.

Future research with larger samples of adolescents from a range of cultural backgrounds and/or with NDDs might result in revised versions of the PALs that comprise fewer items and/or fewer response options, either in total or across the different subscales. 
There was no demonstration of convergent and discriminant validity in the present study. Future research must make this a focus if the 24-item PALs is to be used as an indicator of adolescent loneliness, for monitoring population level changes in loneliness, and as an outcome measure in interventions targeting aspects of mental health.

\section{Clinical Implications}

In conclusion, loneliness is complex and toxic [31] and is associated with worse health outcomes [51], including a 26\% increase in premature mortality [52]. Adolescence is the peak period for loneliness and for over $20 \%$ of adolescents' feelings of loneliness become chronic. The PALs provides clinicians and educators with a measure to assess loneliness, which may assist in identifying potential adverse mental health.

\section{Compliance with ethical standards}

Funding. This work was supported by the Australian Research Council under Grant DP180100252.

Conflict of interest. The authors declare that they have no conflicts of interest.

Availability of data and material. The data set associated with the paper can be obtained from the first author with the agreement of the other authors.

Ethics approval. This paper is based on a secondary analysis of data. The protocol, information sheets, informed consent forms, and other requested documents have been reviewed and approved by The University of Western Australia (RA/4/20/1039) and the Western Australian Department of Education (D18/0207029) with respect to the scientific content and compliance to the applicable health and education regulations. All procedures performed in the study involving human participants were in accordance with the ethical standards of the 1964 Helsinki Declaration and its later amendments or comparable ethical standards.

\section{References}

1. Houghton, S., Hattie, J., Wood, L., Carroll, A., Martin, K., \& Tan, C. (2014).

Conceptualising loneliness in adolescents: Development and validation of a self-report instrument. Child Psychiatry \& Human Development, 45(5), 604-616. 
2. Qualter, P., Vanhalst, J., Harris, R., Van Roekel, E., Lodder, G., Bangee, M., ... \& Verhagen, M. (2015). Loneliness across the life span. Perspectives Psychological Science, 10, 250-264.

3. Lasgaard, M., Armour, C., Bramsen, R.H., \& Goossens, L. (2016). Major life events as predictors of loneliness in adolescence. Journal of Child and Family Studies, 25(2), 631637.

4. Maes, M., Vanhalst, J., Spithoven, A.W., Van den Noortgate, W., \& Goossens, L. (2016). Loneliness and attitudes toward aloneness in adolescence: A person-centered approach. Journal of Youth and Adolescence, 45(3), 547-567.

5. van Dulmen, M.H., \& Goossens, L. (2013). Loneliness trajectories. https://doi.org/10.1016/j.adolescence.2013.08.001

6. Heinrich, L. \& Gullone, E. (2006). The clinical significance of loneliness: A literature review. Clinical Psychology Review, 26,695-718.

7. Qualter, P., Brown, S.L., Rotenberg, K. J., Vanhalst, J., Harris, R.A., Goossens, L., ... \& Munn, P. (2013). Trajectories of loneliness during childhood and adolescence: Predictors and health outcomes. Journal of Adolescence, 36(6), 1283-1293.

8. Gvion, Y., Horresh, N., Levi-Belz, Y., Fischel, T., Treves, I., Weiser, M., .. \& Apter, A. (2014). Aggression-impulsivity, mental pain, and communication difficulties in medically serious and medically non-serious suicide attempters. Comprehensive Psychiatry, 55(1), 40-50.

9. Elmose, M., \& Lasgaard, M. (2017). Loneliness and social support in adolescent boys with attention deficit hyperactivity disorder in a special education setting. Journal of Child and Family Studies, 26(10), 2900-2907. 
10. Lasgaard, M., Nielsen, A., Eriksen, M.E., \& Goossens, L. (2010). Loneliness and social support in adolescent boys with autism spectrum disorders. Journal of Autism and Developmental Disorders, 40(2), 218-226.

11. Whitehouse, A., Durkin, K., Jaquet, E., \& Ziatas, K. (2009). Friendship, loneliness \& depression in Asperger's Syndrome. Journal of Adolescence, 32(2), 309-322.

12. Majorano, M., Brondino, M., Morelli, M., \& Maes, M. (2017). Quality of relationship with parents and emotional autonomy as predictors of self-concept and loneliness in adolescents with learning disabilities: the moderating role of the relationship with teachers. Journal of Child and Family Studies, 26(3), 690-700.

13. Arim, R.G., Kohen, D.E., Garner, R.E., Lach, L.M., Brehaut, J.C., MacKenzie, M.J., \& Rosenbaum, P.L. (2015). Psychosocial functioning in children with neurodevelopmental disorders and externalizing behavior problems, Disability and Rehabilitation, 37(4), 345354.

14. Russell, D. W. (1996). UCLA Loneliness Scale (Version 3): Reliability, validity, and factor structure. Journal of Personality Assessment, 66(1), 20-40.

15. Lee, J., \& Cagle, J.G. (2017). Validating the 11-Item revised university of California Los Angeles scale to assess loneliness among older adults: An evaluation of factor structure and other measurement properties. The American Journal of Geriatric Psychiatry, 25(11), 1173-1183.

16. Robert, E.R., Peter, M.L., \& John, R.S. (1993). A brief measure of loneliness suitable for use with adolescents. Psychological Reports, 72(3 suppl), 1379-1391.

17. Wu, C., \& Yao, G. (2008). Psychometric analysis of the short-form UCLA loneliness scale (ULS-8) in Taiwanese undergraduate students. Personality and Individual Differences, 44, 1762-1771. 
18. Neto, F. (2014). Psychometric analysis of the short-form UCLA loneliness scale (ULS-6) in older adults. European Journal of Ageing, 11, 313-319.

19. Wongpakaran, N., Wongpakaran, T., Pinyopornpanish, M., Simcharoen, S., Suradom, C., Varnado, P., \& Kuntawong, P. (2020). Development and validation of a 6-item Revised UCLA Loneliness Scale (RULS-6) using Rasch analysis. British Journal of Health Psychology, 25(2), 233-256.

20. Mary Elizabeth, H., Linda, J. W., Louise, C. H., \& John, T. C. (2004). A short scale for measuring loneliness in large surveys: Results from two population-based studies. Research on Aging, 26(6), 655-672.

21. de Jong Gierveld, J., \& Kamphuis, F.H. (1985). The development of a Rasch-type loneliness scale. Applied Psychological Measurement, 9, 289-299.

22. Penning, M., Liu, G., \& Chou, P.H.B. (2014).Measuring loneliness among middle-aged and older adults: The UCLA and de Jong Gierveld Loneliness scales. Social Indicators Research, 118(3), 1147-1166.

23. Sancho, P., Pinazo-Hernandis, S., Donio- Bellegarde, M., \& Tomás, J. M. (2020). Validation of the University of California, Los Angeles Loneliness Scale (version 3) in Spanish older population: An application of exploratory structural equation modelling. Australian Psychologist, 55(3), 283-292.

24. Maes, M., Klimstra, T., Van den Noortgate, W., \& Goossens, L. (2015). Factor structure and measurement invariance of a multidimensional loneliness scale: Comparisons across gender and age. Journal of Child and Family Studies, 24(6), 1829-1837.

25. van Roekel, E., Ha, T., Scholte, R.H., Engels, R.C., \& Verhagen, M. (2016). Loneliness in the daily lives of young adults: Testing a socio-cognitive model. European Journal of Personality, 30(1), 19-30. 
26. Goossens, L., \& Beyers, W. (2002). Comparing measures of childhood loneliness: Internal consistency and confirmatory factor analysis. Journal of Clinical Child and Adolescent Psychology, 31(2), 252-262.

27. Goossens, L., Lasgaard, M., Luyckx, K., Vanhalst, J., Mathias, S., \& Masy, E. (2009). Loneliness and solitude in adolescence: A confirmatory factor analysis of alternative models. Personality and Individual Differences, 47(8), 890-894.

28. Marcoen, A., Goossens, L., \& Caes, P. (1987). Loneliness in pre-through late adolescence: Exploring the contributions of a multidimensional approach. Journal of Youth and Adolescence, 16(6), 561-577

29. Maes, M., Van den Noortgate, W., \& Goossens, L. (2015). A reliability generalization study for a multidimensional loneliness scale. European Journal of Psychological Assessment.

30. Houghton, S., Roost, E., Carroll, A., \& Brandtman, M. (2015). Loneliness in children and adolescents with and without attention-deficit/hyperactivity disorder. Journal of Psychopathology and Behavioral Assessment, 37(1), 27-37.

31. Houghton, S., Hattie, J., Carroll, A., Wood, L., \& Baffour, B. (2016). It hurts to be lonely! Loneliness and positive mental wellbeing in Australian rural and urban adolescents. Journal of Psychologists and Counsellors in Schools, 26(1), 52-67.

32. Andrich, D. (2011) Rating scales and Rasch measurement. Expert Review of Pharmacoeconomics \& Outcomes Research, 11(5), 571 - 585.

33. Rasch, G. (1960). Probabilistic models for some intelligence and attainment tests. Copenhagen: Danish Institute for Educational Research.

34. Rasch, G. (1980). Probabilistic models for some intelligence and attainment tests (Expanded.). Chicago: University of Chicago Press. 
35. Zaporozhets, O., Fox, C.M., Beltyukova, S.A., Laux, J.M., Piazza, N.J., \& Salyers, K. (2015). Refining change measure with the Rasch model. Measurement and Evaluation in Counseling and Development, 48(1), 59-74.

36. Tennant, A., McKenna, S.P., \& Hagell, P. (2004). Application of Rasch analysis in the development and application of quality of life instruments. Value in Health, 7, S22-S26.

37. APA (2000). Diagnostic and Statistical Manual of Mental Disorders, 4th edn, text revision (DSMIV-TR). Washington DC: APA.

38. Andrich, D. (2009) Using the Rasch Unidimensional Measurement Model (RUMM2030) software.

39. Andrich, D., Sheridan, B., \& Luo, G. (2018). RUMM2030: Rasch unidimensional models for measurement. Perth UWA, Australia: RUMM Laboratory

40. Andrich, D. (1982). An index of person separation in latent trait theory, the traditional KR.20 index, and the Guttman scale response pattern. Education Research and Perspectives, 9(1), 95-104.

41. Andrich, D. \& Marais, I. (2019). A course in Rasch Measurement Theory: Measuring in the Educational, Social and Health Sciences. Springer, Singapore.

42. Smith, E. (2005). Effect of item redundancy on Rasch item and person estimates. Journal of Applied Measurement 6, 147-163.

43. Cameron, I.M., Scott, N.W., Adler, M., Reid, I.C. (2014). A comparison of three methods of assessing differential item functioning (DIF) in the Hospital Anxiety Depression Scale: ordinal logistic regression, Rasch analysis and the Mantel chi-square procedure. Quality of Life Research, 23, 2883 - 2388.

44. Linacre, J.M. (2002). Optimizing rating scale category effectiveness. Journal of Applied Measurement, 3(1), 85-106.

45. Bond, T. G., \& Fox, C. M. (2015). Applying the Rasch model: Fundamental measurement in the human sciences (3rd ed.). New York, NY: Routledge. 
46. Meijer, R.R., \& Egberink, I.J. (2012). Investigating invariant item ordering in personality and clinical scales: Some empirical findings and a discussion. Educational and Psychological Measurement, 72(4), 589-607

47. King-Dowling, S., Proudfoot, N. A., \& Obeid, J. (2019). Comorbidity among chronic physical health conditions and neurodevelopmental disorders in childhood. Current Developmental Disorders Reports, 6(4), 248-258.

48. Capodieci, A., Crisci, G., \& Mammarella, I.C. (2019). Does positive illusory bias affect self-concept and loneliness in children with symptoms of ADHD? Journal of Attention Disorders, 23(11), 1274-1283.

49. Macdonald, S.J., Deacon, L., Nixon, J., Akintola, A., Gillingham, A., Kent, J., ... \& Dore, S. (2018). ‘The invisible enemy’: disability, loneliness and isolation. Disability \& Society, 33(7), 1138-1159.

50. Lykes, V.A., \& Kemmelmeier, M. (2014). What predicts loneliness? Cultural difference between individualistic and collectivistic societies in Europe. Journal of Cross-Cultural Psychology, 45, 468-490.

51. Luo, Y., Hawkley, L.C., Waite, L.J., \& Cacioppo, J.T. (2012). Loneliness, health, and mortality in old age: A national longitudinal study. Social Science \& Medicine, 74(6), 907914.

52. Cacioppo, J.T., \& Cacioppo, S. (2018). The growing problem of loneliness. The Lancet, 391(10119), 426. 


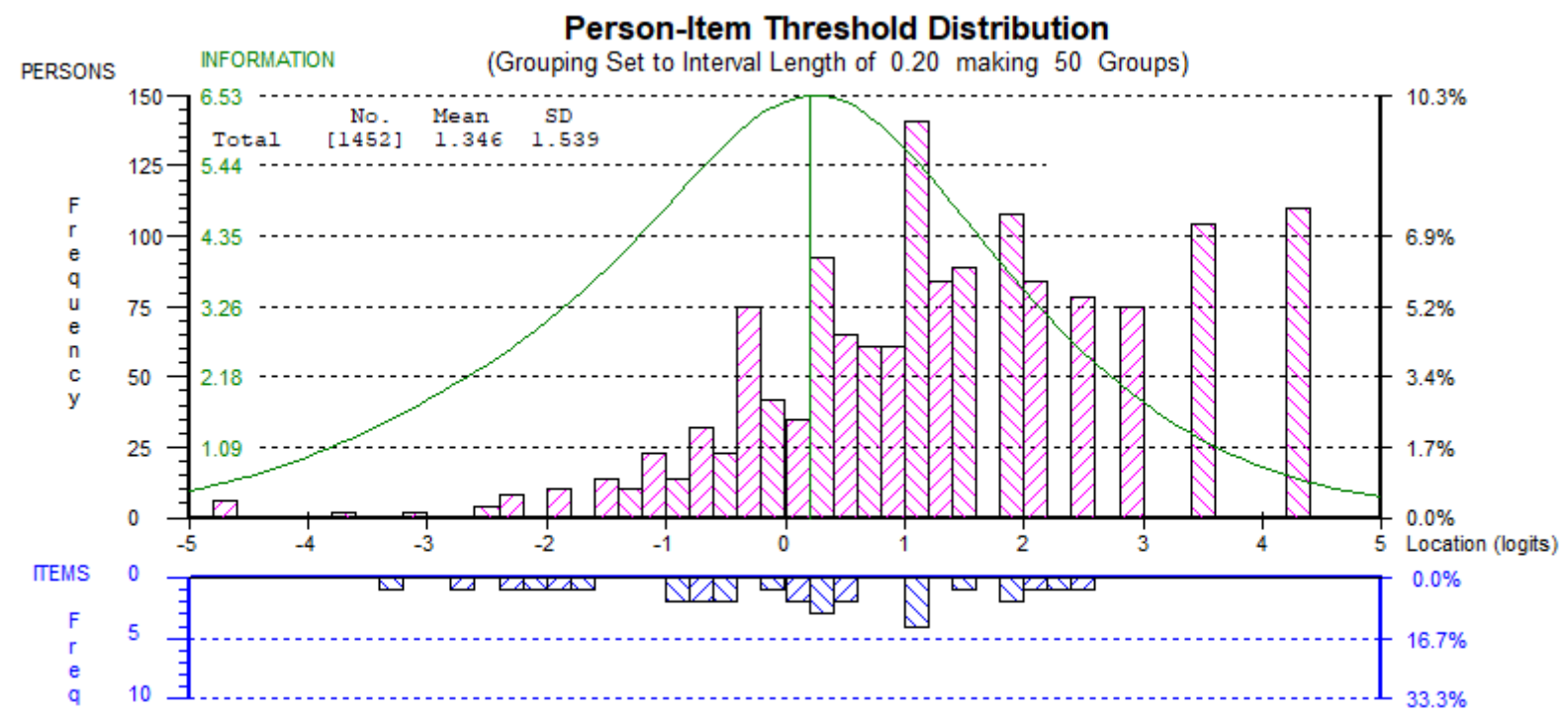

The information function (indicated on the graph in green) shows that friendship-related loneliness is most accurately estimated for those with mid-range scores, rather than very high or low scores.

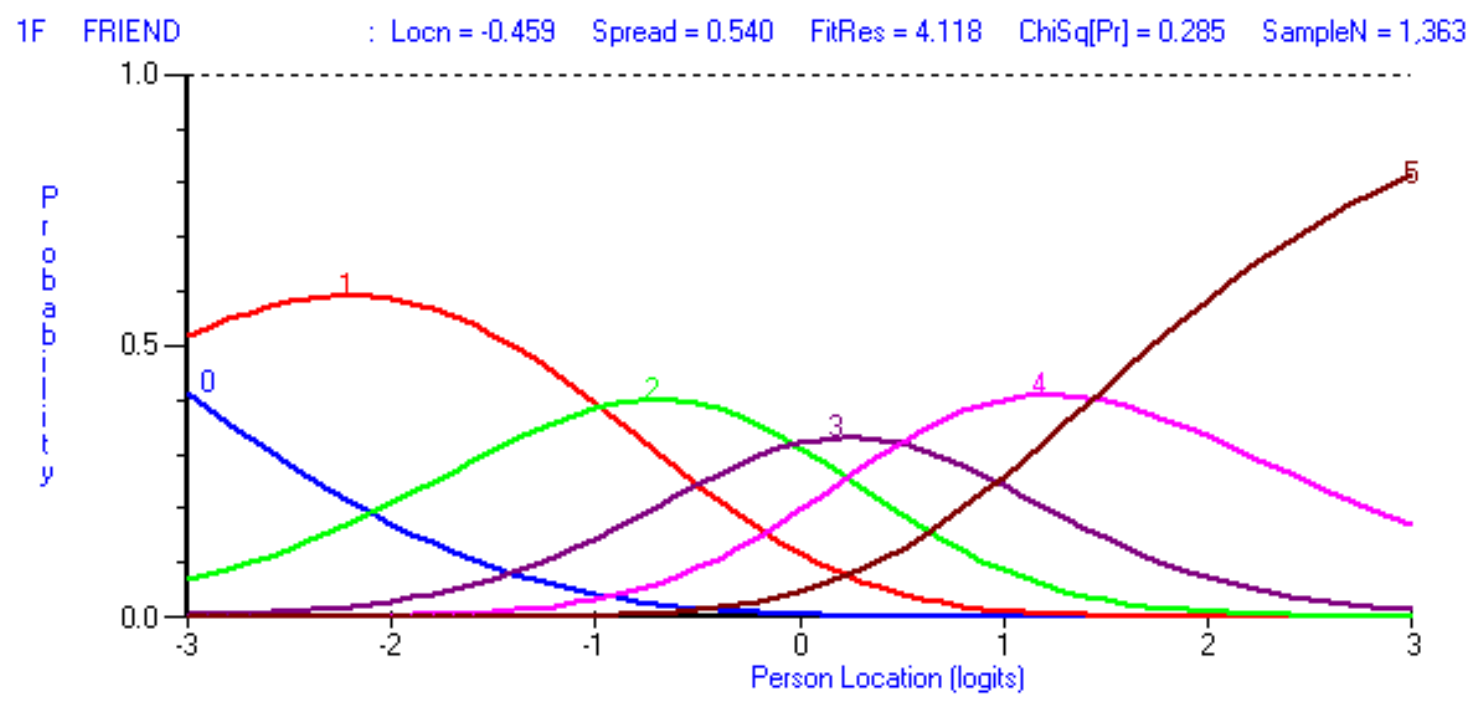

Figure 1 Friendship Related Loneliness subscale: Histograms of person and item threshold estimates (top) and Category Characteristic Curves (CCC) for item 1 (I feel part of a group of friends) (bottom). 


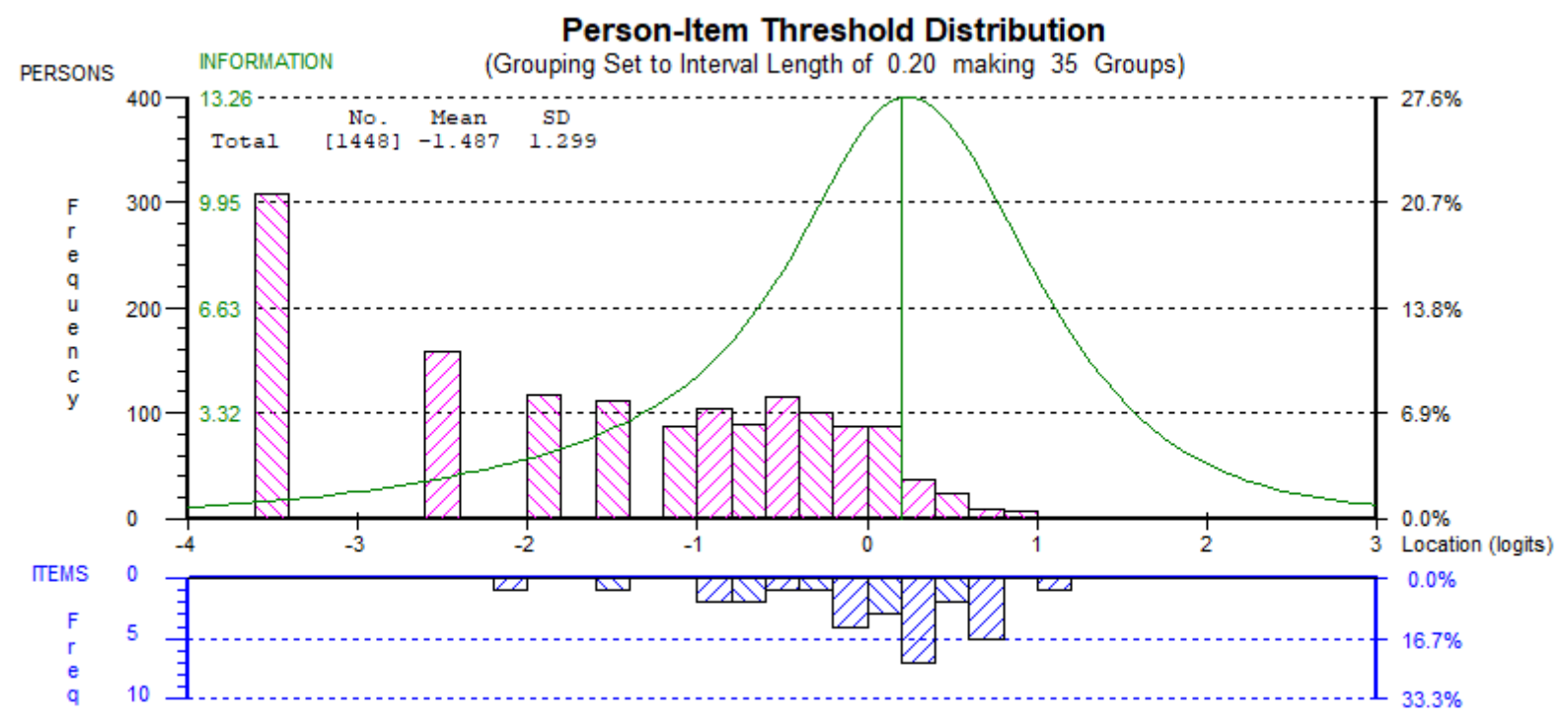

The information function (indicated on the graph in green) shows that isolation is most accurately estimated for those with mid-range scores, rather than lower scores.

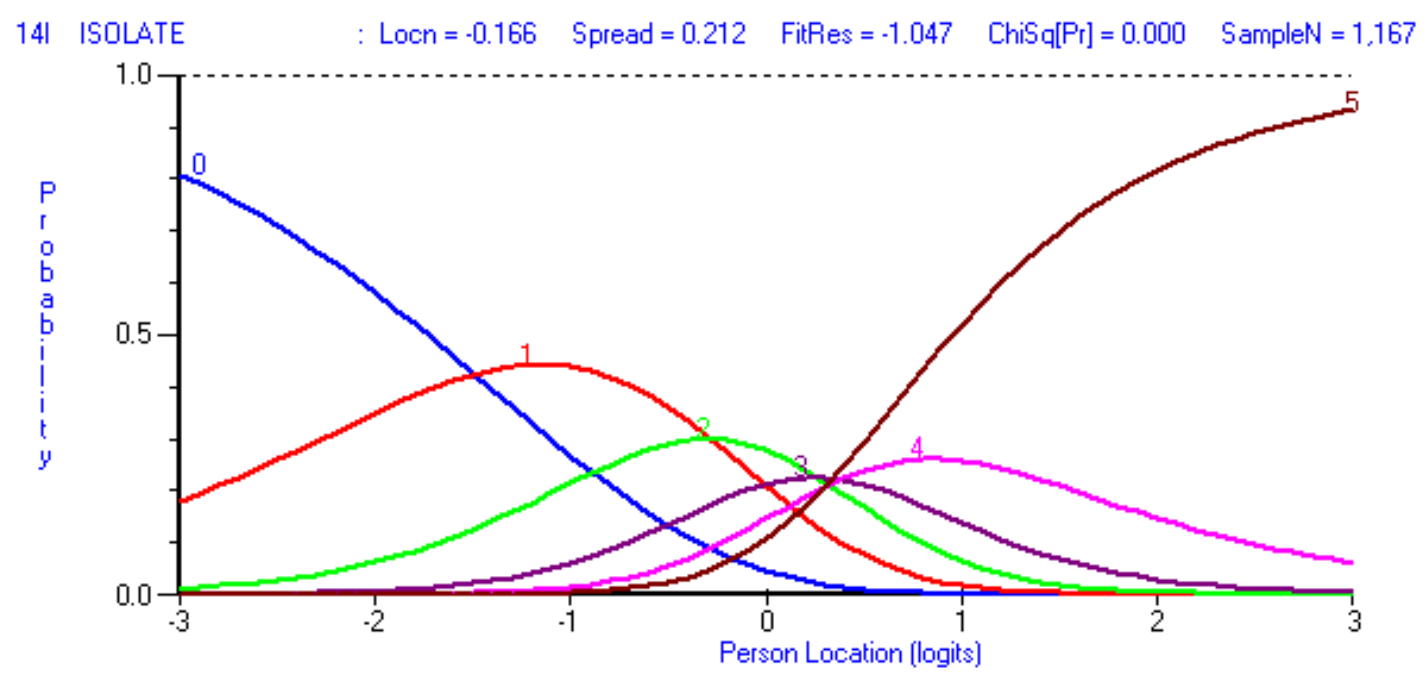

Figure 2 Isolation subscale: Histograms of person and item threshold estimates (top) and Category Characteristic Curves for item 14 (No one cares much about me) (bottom). 


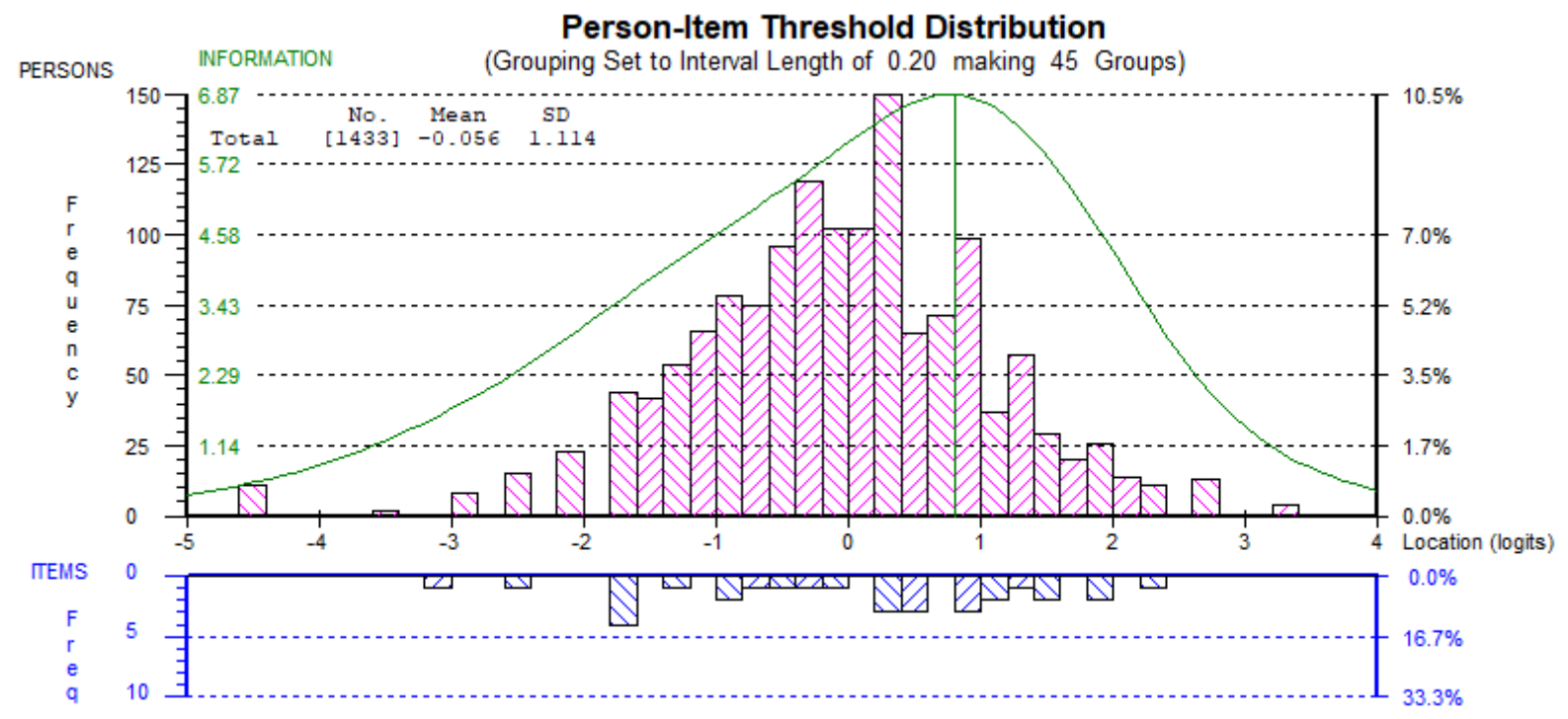

The information function (indicated on the graph in green) shows that positive attitude to aloneness is most accurately estimated for those with moderate to higher scores on the scale.

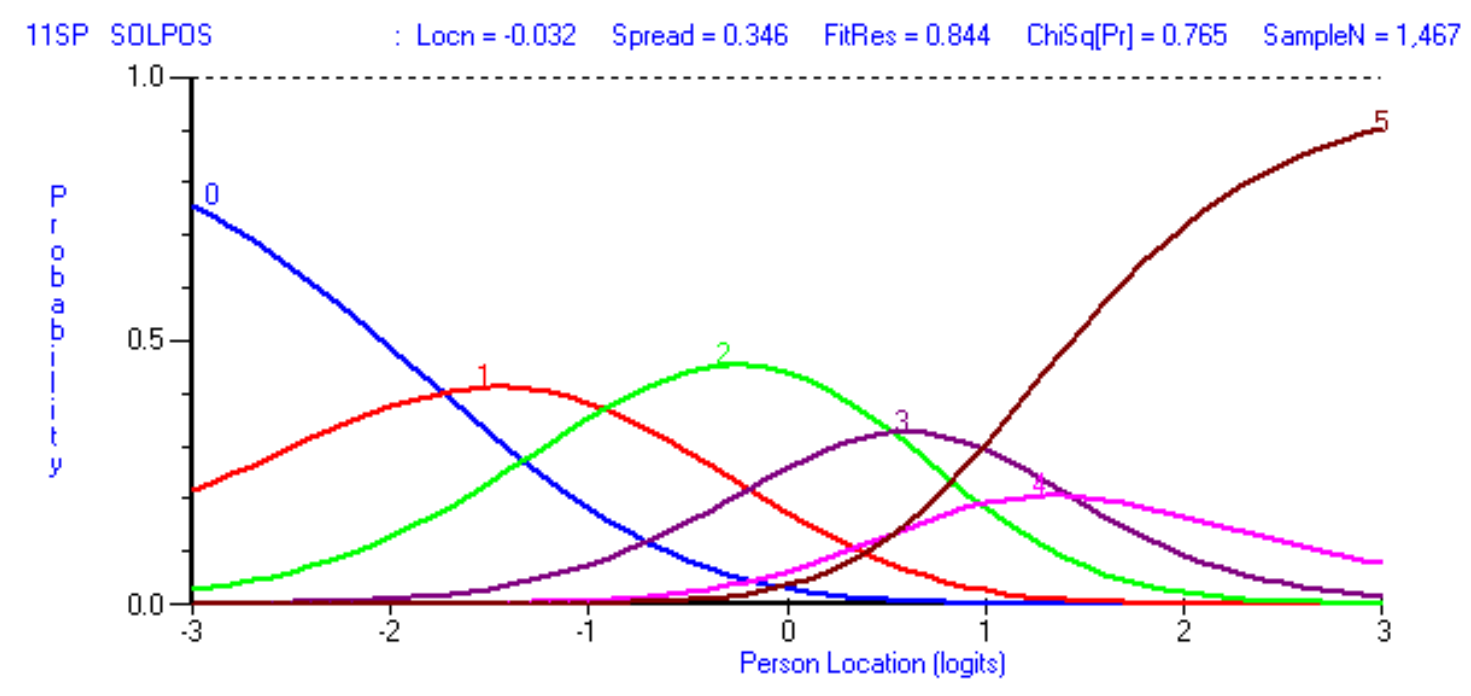

Figure 3 Positive Attitude to Aloneness subscale: Histograms of person and item threshold estimates (top) and Category Characteristic Curves for item 11 (There are positive things about being alone) (bottom). 


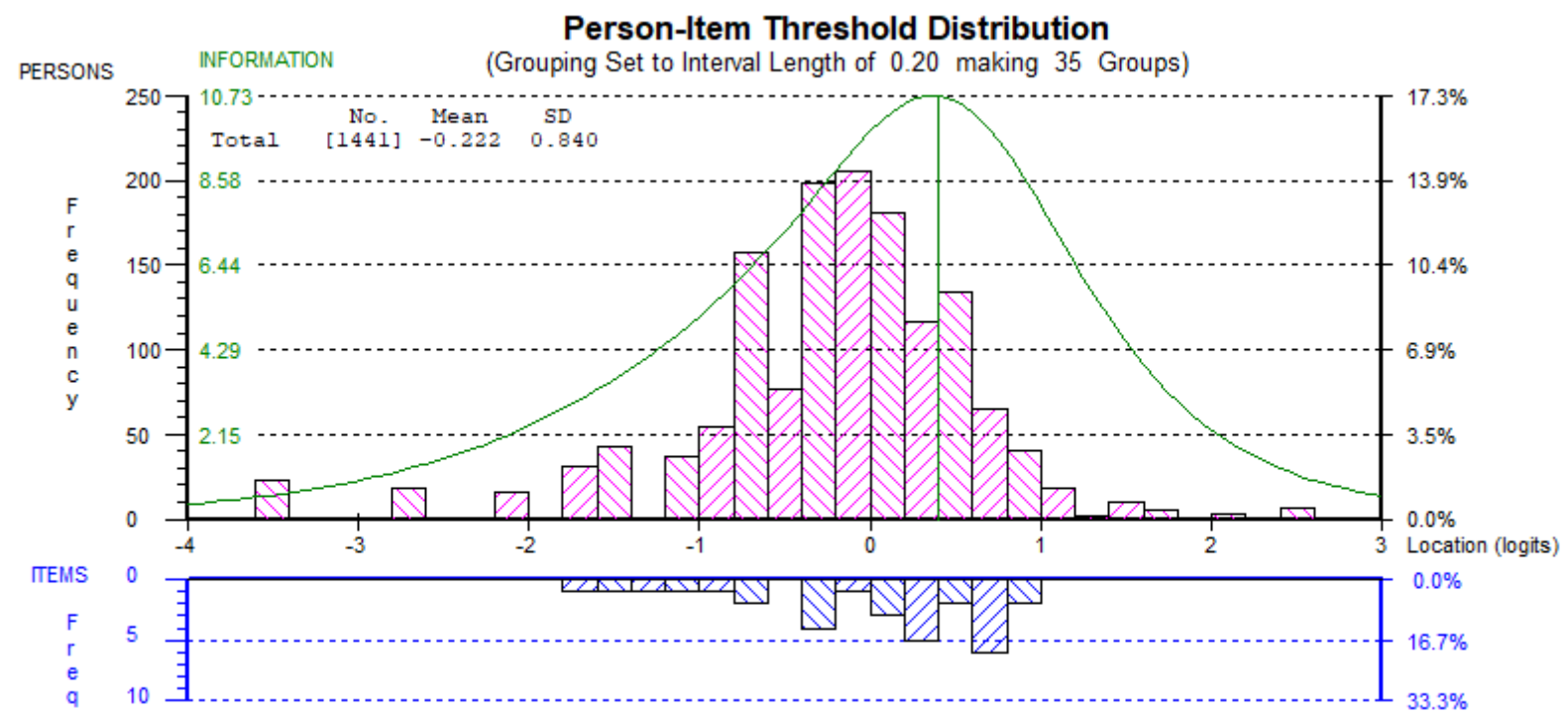

The information function (indicated on the graph in green) shows that negative attitude to aloneness is most accurately estimated for those with moderate to higher scores on the scale.

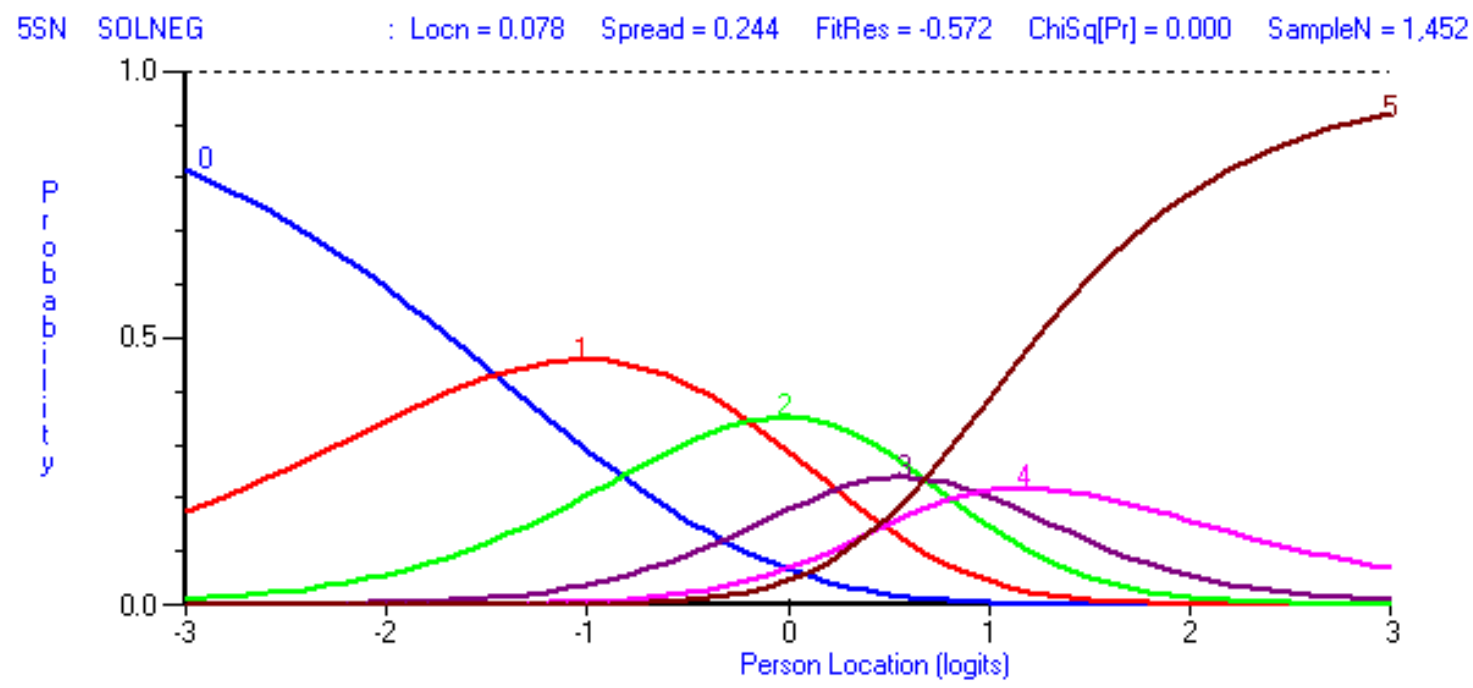

Figure 4 Negative Attitude to Aloneness subscale: Histograms of person and item threshold estimates (top) and Category Characteristic Curves for item 5 (When I am by myself I feel lonely) (bottom). 


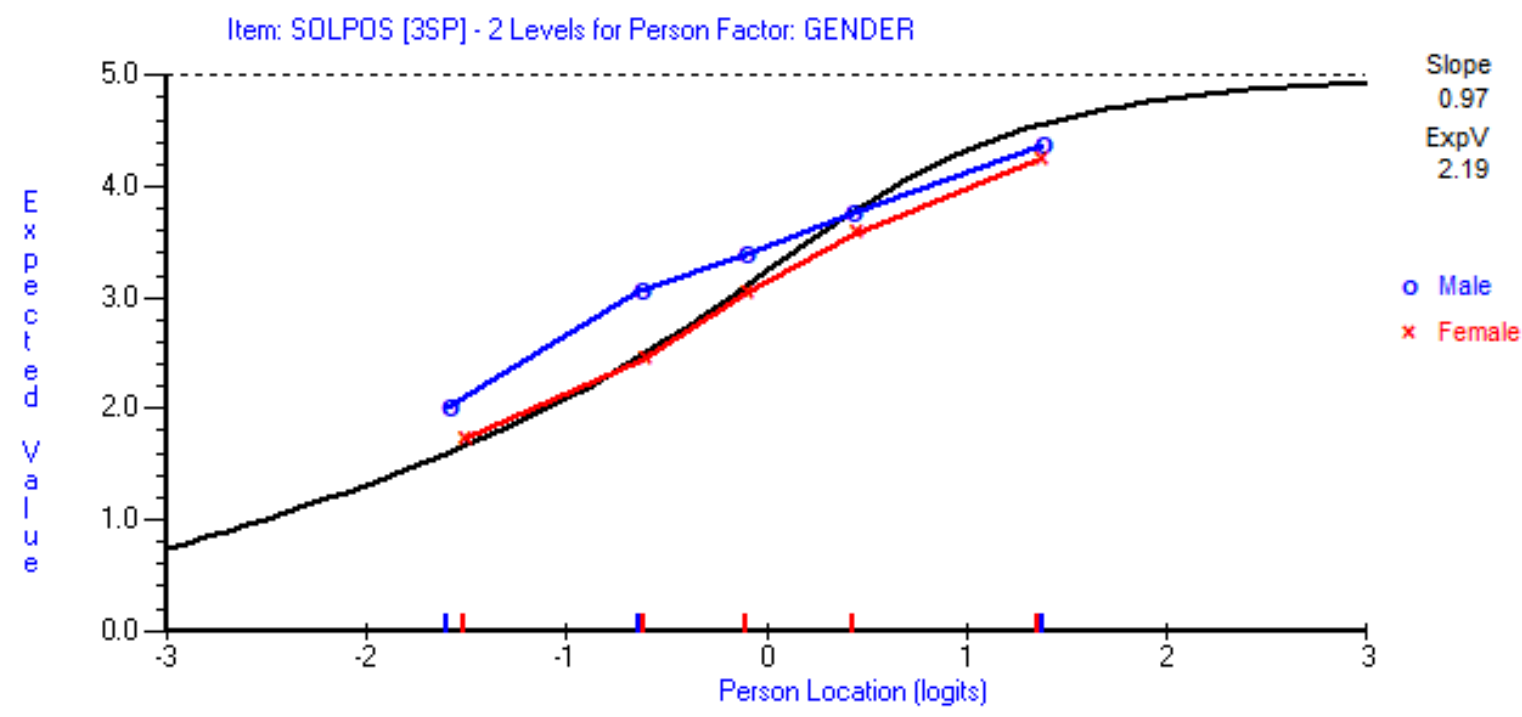

Figure 5 DIF for gender for Item 3 (I feel calm when I'm by myself) on the Positive Attitude to Aloneness subscale. 


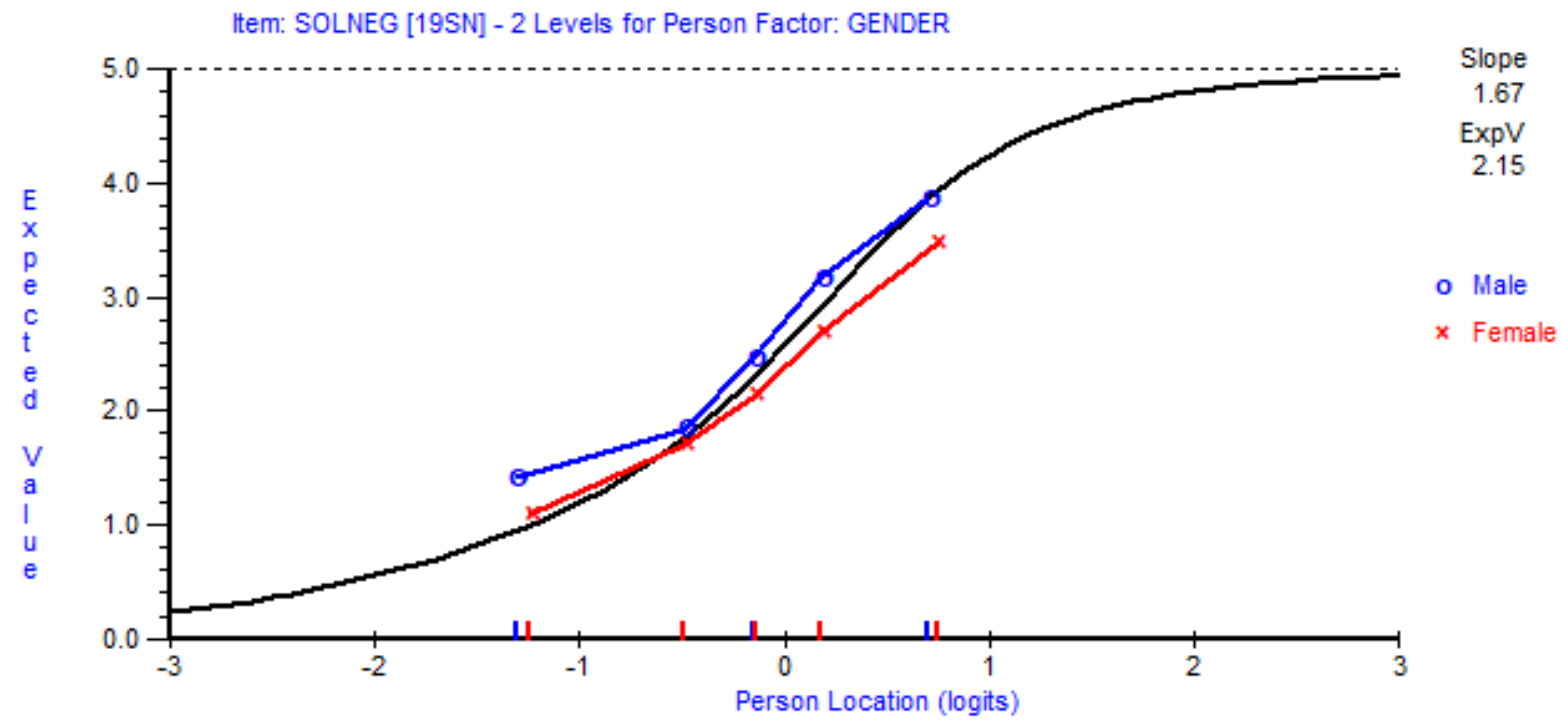

Figure 6 DIF for gender for Item 19 (when I get bored I'm unhappy) on the Negative Attitude to Aloneness subscale. 